\title{
UNUSUAL PRESENTATION OF ACUTE PULMONARY EDEMA IN SEVERE PREECLAMPTIC PATIENT DURING CESAREAN SECTION: A CASE REPORT.
}

Habib Md Reazaul Karim, Jayanta Kr. Mitra, Prithwis Bhattacharyya, Amy G. Rapsang
1. Post Graduate Trainee, Department of Anaesthesia, NEIGRIHMS, Shilong. Meghalaya.
2. Assistant Professor, Department of Anaesthesia, NEIGRIHMS, Shilong. Meghalaya.
3. Professor \& Head, Department of Anaesthesia, NEIGRIHMS, Shilong. Meghalaya.
4. Senior Resident, Department of Anaesthesia, NEIGRIHMS, Shilong. Meghalaya.

\section{CORRESPONDING AUTHOR}

Dr. Jayanta Kr. Mitra,

Department of Anaesthesiology, NEIGRIHMS, Mawdiangdiang, Shillong,

E-mail: mitra.jayanta@gmail.com,

Ph: 00919402507025.

\begin{abstract}
Acute pulmonary edema during pregnancy is very rare and occurs in $0.08 \%$ pregnancies. About 3\% of severe preeclamptic patients develop acute pulmonary edema. Among them $30 \%$ develop it in the antenatal period with $90 \%$ having pre existing chronic hypertension and $70 \%$ develop it in the postnatal period. Several risk factors have been identified: preeclampsia or eclampsia, use of tocolytic therapy, severe infection, cardiac disease, iatrogenic fluid overload, and multiple gestations. No matter what the underlying pathology, resuscitation is the foremost priority. Only after the patient has been stabilized, attention can be turned to the formulation of a differential diagnosis to address the underlying condition and initiate specific treatment. This case examines one such presentation and reviews some of the diagnostic possibilities.
\end{abstract}

KEY WORDS: Pregnancy, severe preeclampsia, pregnancy and obesity, acute pulmonary edema, acute pulmonary edema in preeclampsia, emergency cesarean section, complications in pregnancy

INTRODUCTION: Acute pulmonary edema during pregnancy, though an uncommon event in pregnancy $(0.08 \%)^{\cdot[1]}$ is associated with an increased risk of maternal and fetal morbidity and mortality. Pulmonary edema is infrequently encountered in patients with severe pre-eclampsia without associated medical, surgical or obstetric complications. In an analytical study of 37 consecutive cases by Sibai et al.[2] pulmonary edema was diagnosed in $2.9 \%$ of cases complicated by severe pre-eclampsia and eclampsia. One such case is reported which is presented unusually during the time of cesarean section.

CASE REPORT: A 27 year old, booked primigravida with single live intrauterine fetus with gestational age of 39 weeks presented to labor room with complaints of sudden onset blurring of vision, headache, and nausea since morning. Patient had history of mild degree high blood pressure (140/90-150/90 $\mathrm{mmHg}$ ) for last 1 month but was not on any medication. On the day she presented to our hospital with headache and BP of 190/120 mmHg with urine showed albumin (++) and pedal edema. Inj. Labetalol $20 \mathrm{mg}$ iv and inj. MgSO4 $10 \mathrm{gm}$ IM loading dose was given. Cardiotocogram showed reactive pattern with baseline HR between 150-155 beats per minutes. Patient's condition was deteriorating clinically and blood pressure was not 
responding to even after three repeated doses of injection Labetalol $10 \mathrm{mg}$ each at an interval of 5 to 10 minutes. Emergency LSCS was planned and patient shifted to operating room. Patient was conscious alert and oriented, afebrile, morbidly obese (BMI $41.15 \mathrm{~kg} / \mathrm{m} 2$ ) with weight of $108 \mathrm{~kg}$ and her estimated ideal body weight was $54.7 \mathrm{Kg}$. Patient gave no history of smoking, tobacco or any drug abuse. No other co morbid conditions were found.

She took her last meal $2 \mathrm{hrs}$ ago. General physical examination revealed BP 186/126 $\mathrm{mmHg}$ and heart rate 124 /minute, regular, respiratory rate 24/ minute and bilateral pitting pedal edema. Bilaterally chest was clear, with normal vesicular breath sound with no adventitious sounds. Both the heart sounds heard, were normal and no added sounds. No neurological deficit was found. Fetal heart rate was 148/min.

Airway examination revealed adequate mouth opening with inter incisor distance of more than 3 finger width; she had no dentures, artificial or loose teeth. Flexion and extension of neck was normal.Neck was thick and short with thyromental distance $6 \mathrm{~cm}$ and sternomental distance $10 \mathrm{~cm}$ approximately and Mallampati class -II.

Patient was kept on left lateral position in pre-op area with Oxygen supplementation by face mask. Through an 18 gauge IV line in left forearm, colloid (Hydroxy ethyl starch 130/0.4) was started. Patient was premedicated with Inj. Metoclopramide $10 \mathrm{mg}$ and inj. Ranitidine 50mg (non particulate antacid not available) and shifted to operating room with prepared difficult intubation cart.

Patient positioned with wedge under right buttock, oxygen supplementation continued and monitoring connected. General anesthesia planned on view of unknown coagulation profile. Procedure explained including cricoid pressure to the patient. Injection Labetalol $20 \mathrm{mg}$ IV bolus was given. Preoxygenation was done for 5 minutes. After dressing and draping rapid sequence induction started along with cricoid pressure with Inj Thiopentone 500mg and Inj Succinylcholine $100 \mathrm{mg}$.Ventilation was done with appropriate size guedel's airway but patient desaturated to $85 \%$ within $15-20$ seconds of Succinylcholine injection. An intubation trial was done and was successful in first attempt with $7 \mathrm{~mm}$ ID size endotracheal tube under direct vision, position confirmed with capnography, tube cuff inflated and cricoid pressure released.

Patient was ventilated in volume control mode with 100\% oxygen and PEEP $5 \mathrm{~cm} \mathrm{H}_{2} \mathrm{O}$ but the saturation did not improve. When the PEEP was increased to $10 \mathrm{~cm}$ of $\mathrm{H} 20$, the saturation improved to $95 \%$ and remained there till delivery, after which it improved to $100 \%$.

After 10 min of intubation- white frothy secretion was noted in ET tube, but there was no particulate material or blood. Saturation came down to $88 \%$. Intermittent suctioning of tube was performed to maintain saturation above $95 \%$ with $100 \%$ oxygen. Inj.Frusemide $20 \mathrm{mg}$ IV was given suspecting pulmonary oedema.

General anesthesia maintained with Isoflurane (MAC 0.65 to 0.70 ) and after delivery Inj. Fentanyl $60 \mathrm{mcg}$, Inj. Midazolam $2 \mathrm{mg}$ and inj. Ketorolac $30 \mathrm{mg}$ used. Inj. Oxytocin 10 units were added in drip after delivery and uterine contraction was found to be adequate. Baby was shifted to NICU, after resuscitation and evaluation by attending pediatrician.

Intraoperative systolic BP had varied between 140-150 mmHg and diastolic BP between $90-100 \mathrm{mmHg}$ ) and heart rate remained close to baseline. IV Fluid $500 \mathrm{ml}$ of colloid and $700 \mathrm{ml}$ Ringer Lactate over 1 hour was given intraoperatively.

Postoperatively patient was unable to maintain saturation on manual bag ventilation and blood pressure reached 180/120 $\mathrm{mmHg}$, so anesthesia deepened and patient shifted to ICU with Bain's circuit with application of PEEP without extubation. Shifting was uneventful. In the ICU the patient was put on SIMV mode with PEEP $10 \mathrm{H}_{2} \mathrm{O}$. Blood pressure was controlled with 
NTG infusion @ $0.5 \mathrm{mcg} / \mathrm{kg} /$ minute. Inj. Frusemide infusion started @ 20 mg/hour. MgSO4 was not given intraop and immediate post operative period because patient was under GA and facility for estimation of serum Mg level was not available. Patient was recovering well and blood pressure under control. She was weaned from ventilator same night and kept on T-piece and extubated in the morning next day. NTG infusion tapered off same night and patient did not need any further antihypertensive.

Patient needed oxygen supplementation for 24 hour postoperatively. She was shifted to ward with advice to perform deep breathing exercise and incentive spirometry.

DISCUSSION: Acute pulmonary edema is a relatively rare complication during pregnancy. A review of 62,917 consecutive pregnancies by Sciscione et al. ${ }^{[2]}$ has shown that the incidence acute pulmonary edema during pregnancy or in the postpartum period is $0.08 \%$.

Pulmonary edema is defined as the accumulation of fluid in the pulmonary interstitial spaces and alveoli, which prevents the adequate diffusion of both oxygen and carbon dioxide. The pathogenesis of pulmonary edema complicating pre-eclampsia-eclampsia is a subject of extensive investigation. The low colloid oncotic pressure, coupled with increased vascular permeability and the loss of intravascular fluid and protein into interstitial tissues, increases the risk of pulmonary edema. ${ }^{[3]}$ Preeclampsia is a multisystem disorder that primarily affects the maternal cardiovascular, central nervous, and genitourinary systems. However, all systems become involved to some degree. Acute pulmonary edema is a severe, sometimes frightening complication of severe preeclampsia and eclampsia. A report by Donnelly and Lock ${ }^{[4]}$ on 533 patients who died of toxemia showed that pulmonary edema was a cause of death in $25 \%$ cases. Pregnancy decreases oxygen reserves secondary to decrease in the functional residual capacity. Obesity leads to fat accumulation on the thorax and abdomen which decreases chest wall and lung compliance. Decreased pulmonary compliance leads to decreased functional residual capacity (FRC), vital capacity, and total lung capacity. Reduced FRC can result in lung volumes below closing capacity in the course of normal tidal ventilation, leading to small airway closure, ventilation-perfusion mismatch, right-to-left shunting, and arterial hypoxemia. Anesthesia worsens this situation so much so that up to a $50 \%$ reduction in FRC occurs in the obese anesthetized patient. In this patient the reduced FRC further aggravates the hypoxia in the background of pulmonary edema. Hypoxemia and fall in oxygen saturation are not uncommon during general anaesthesia and any fall in saturation should be taken seriously. After ruling out disturbances of inspired oxygen concentration and ventilation, pulmonary edema should be suspected and other causes such as anemia, capillary leak syndrome and reactions to colloids should also be considered.

\section{REFERENCE:}

1. Sciscione AC, Ivester T, Largoza M, et al: Acute pulmonary edema in pregnancy. Obstet Gynecol 2003; 101:511-515.

2. Sibai BM, MabieWC,Harvey CJ, Gonzalez AR. Pulmonary edema in severe preeclampsiaeclampsia: Analysis of 37 consecutive cases. Am J Obstet Gynecol 1987; 156:1174-9.

3. Benedetti TJ, Kates R, Williams V.: Hemodynamic observations in severe preeclampsia complicated by pulmonary edema. Am J Obstet Gynecol 1985; 152:330-334

4. Donnelly, J. F. and Lock, F. R.: Causes of death in five hundred thirty-three fatal cases of toxemia in pregnancy. Amer. T. Obstet. \& Gynaecol., 86: 184-190, 1954. 


\section{CASE REPORT}

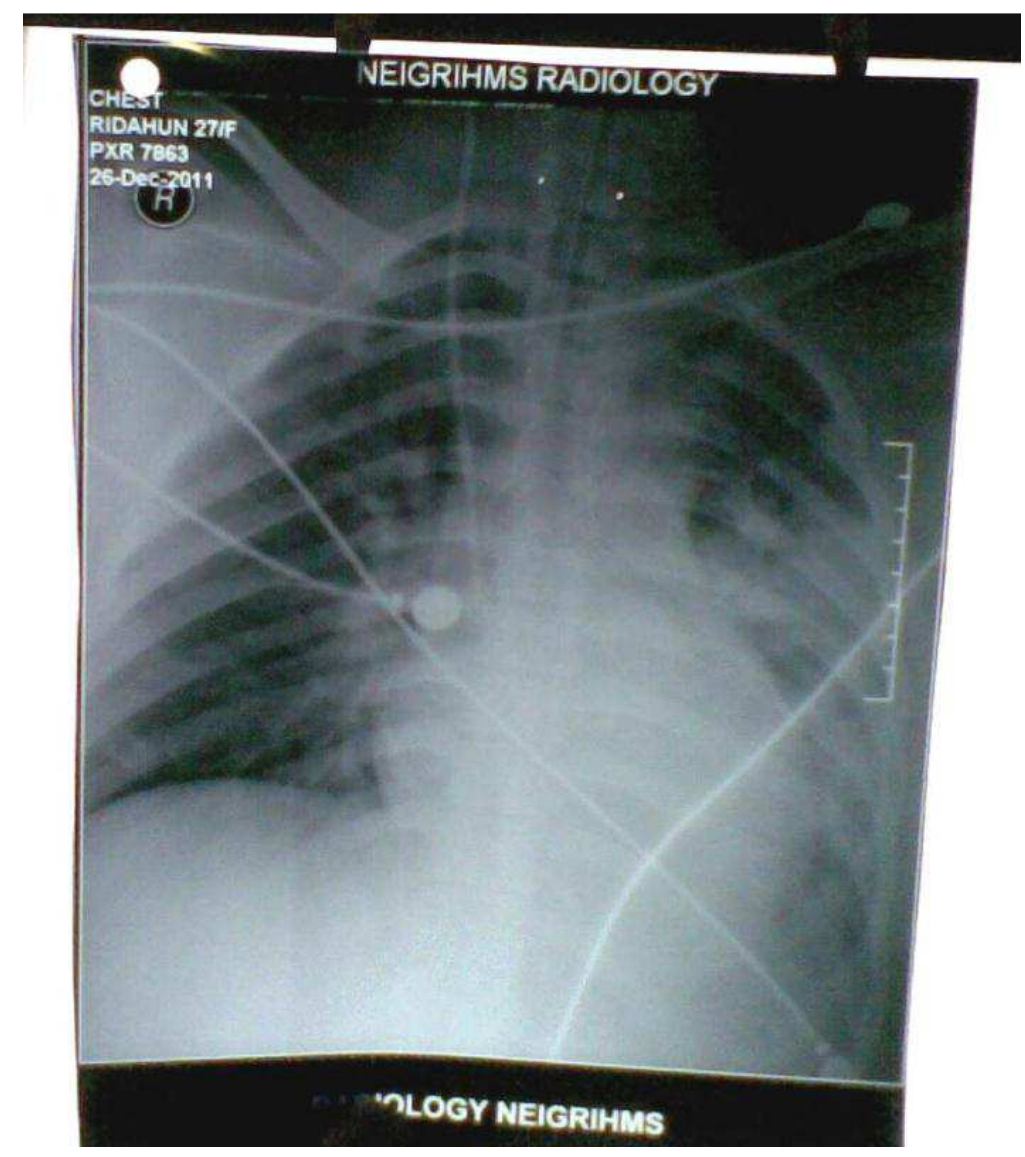

Fig: picture showing pulmonary edema feature in immediate postoperative period. 